\title{
RESEARCH
}

Open Access

\section{Lymphoid enhancer-binding factor 1 (LEF- 1): a favorable prognostic factor in adult acute myeloid leukemia in Egyptian patients}

Botheina Ahmed Thabet Farweez ${ }^{* *}$ (D) Nahela Ahmed Shalaby', Doaa Ahmed Gamal Eissa?', Raghda El Sayed Abdel Monem Galal', Nashwa El-khazragy ${ }^{2}$ and Shaimaa Abdelmalik Pessar ${ }^{1}$

\begin{abstract}
Background: Canonical wingless-type (Wnt) signaling is a crucial pathway involved in normal hematopoiesis and the self-renewal process of hematopoietic stem cells. Deregulation of this pathway has been associated with different subtypes of leukemia. Lymphoid enhancer-binding factor 1 (LEF-1) is a major transcription factor of this pathway and plays a pivotal role in lymphoid differentiation and granulopoiesis. High LEF-1 expression has been reported as a prognostic marker in several types of adult hematological malignancies. We aimed to assess the prognostic utility of LEF-1 expression in adult de novo acute myeloid leukemia (AML) Egyptian patients in continuation of our previous work. LEF-1 expression was analyzed by real-time polymerase chain reaction (PCR) in 30 adults with newly diagnosed AML and remeasured at day 28 after induction therapy with the assessment of remission status.
\end{abstract}

Results: Patients were classified according to median expression level into high and low LEF-1 expression groups. LEF-1 levels were dramatically decreased following successful induction therapy. Also, high LEF-1 expression patients had a better response to therapy with better overall survival. ROC curve analysis of LEF-1 expression yielded a cutoff value of $\leq 10.11 \log _{10}$ (sensitivity of $90.48 \%$ and specificity of 100\%) for predicting poor outcome. Univariate logistic regression analysis showed that for every $\log _{10}$ increase in the LEF-1 expression level, the chance of the patient to achieve hematological remission was increased by 2.29 folds.

Conclusion: Our study showed preliminary results that overexpression of LEF-1 is a favorable prognostic factor in newly diagnosed adult AML patients. The prognostic value of LEF-1 could suggest its utility for further risk classifications of AML and potentiality for being a target for therapy.

Keywords: Adult acute myeloid leukemia, LEF-1 expression, Favorable outcome, Lymphoid enhancer-binding factor 1, Prognostic factor

\footnotetext{
* Correspondence: dr_botheina@hotmail.com

${ }^{1}$ Clinical and Chemical Pathology, Hematology unit, Faculty of Medicine, Ain

Shams University, Cairo, Egypt

Full list of author information is available at the end of the article
}

\section{Springer Open}

(c) The Author(s). 2020 Open Access This article is licensed under a Creative Commons Attribution 4.0 International License, which permits use, sharing, adaptation, distribution and reproduction in any medium or format, as long as you give appropriate credit to the original author(s) and the source, provide a link to the Creative Commons licence, and indicate if changes were made. The images or other third party material in this article are included in the article's Creative Commons licence, unless indicated otherwise in a credit line to the material. If material is not included in the article's Creative Commons licence and your intended use is not permitted by statutory regulation or exceeds the permitted use, you will need to obtain permission directly from the copyright holder. To view a copy of this licence, visit http://creativecommons.org/licenses/by/4.0/. 


\section{Background}

Acute myeloid leukemia (AML) is a disorder with clinical and biological heterogeneity. Therefore, it is always a field of interest to find more markers to optimize the classification, improve the initial risk assessment, and to offer guidance for appropriate treatment.

Lymphoid enhancer-binding factor 1 (LEF-1) is a transcription factor of the Wingless-type (Wnt) $/ \beta$ catenin signaling pathway which controls the proliferation, survival, and differentiation of hematopoietic cells through its effect on target genes like c-myc, cyclin D1 [1]. In normal human hematopoiesis, $L E F-1$ plays a pivotal role not only in the development of $\mathrm{B}$ and $\mathrm{T}$ lymphocytes but also in granulopoiesis. In fact, in healthy individuals, $L E F-1$ mRNA levels reach a maximum at the promyelocytic stage of differentiation and decline during the last steps of granulocyte maturation [2]. Physiologically, the pathway is strictly regulated [3]. In healthy cells, secreted and/or intracellular located inhibitory proteins tightly control $\beta$ catenin levels [4].

Wnt signaling pathway aberrations has been implicated in leukemic transformation and has been shown to promote proliferation and survival of leukemic cells in vitro [5].

The LEF-1 was found to be highly expressed in different hematologic malignancies, including lymphomas and chronic lymphocytic leukemia (CLL) $[6,7]$ where $L E F-1$ showed to be a sensitive and specific marker for CLL and had a diagnostic utility in challenging small B cell lymphoma cases. In acute lymphoblastic leukemia (ALL) [5, 8], high $L E F-1$ expression in B-ALL was a poor prognostic factor and an indicator of a high risk of treatment failure, and in T-ALL [9], LEF-1 inactivation was part of the underlying molecular pathogenesis, whereas in AML [10], $L E F-1$ was found to play a role in the pathophysiology and could serve as a novel predictor of better treatment response. Moreover, some studies suggested that LEF-1 might serve as a target of anti-tumor therapy [4].

Consequently, we aimed to further evaluate the prognostic significance and utility of $L E F-1$ expression in adult de novo AML Egyptian patients as a continuation of our previous work [11] where LEF-1 expression was significantly higher in AML patients compared to controls and showed a positive correlation with hemoglobin $(\mathrm{Hb})$ level and platelet (Plt) count, while negative correlation with age, total leucocytic count (TLC), and peripheral blood blast percentage.

\section{Methods}

This study included 30 newly diagnosed adult de novo AML patients admitted to and followed up for 1 year at the clinical hematology-oncology unit in the period from May 2017 to January 2019. AML patients' diagnosis, management, and follow-up were performed according to the World Health Organization (WHO) Classification of Tumours of Haematopoietic and Lymphoid Tissues
[12] and European Society for Medical Oncology (ESMO) Clinical Practice Guidelines for diagnosis, treatment, and follow-up [13].

Informed consent was obtained from all participant individuals. The study was conducted following the stipulations of the local ethical and scientific committees, and the procedures respected the ethical standards in the Helsinki declaration of 1964.

Induction therapy All patients except AML-M3 (acute promyelocytic leukemia) (APL) were subjected to induction chemotherapy by standard-dose cytarabine plus anthracyclin $(7+3)$ : Ara-c $100 \mathrm{mg} / \mathrm{m}^{2}$ for 7 days and Daunorubicin 60 $\mathrm{mg} / \mathrm{m}^{2}$ for 3 days. APL patients were treated by all-transretinoic acid (ATRA), and in few patients, arsenic trioxide (ATO) was added.

Consolidation therapy For patients achieving remission, high-dose cytarabine (ara-C) (HiDAC) was used.

Refractory cases were treated by either HAM (high-dose cytarabine and mitoxantrone) or FLAG (fludarabine, cytarabine, and G-CSF) protocol.

AML patients' characteristics were evaluated at diagnosis by the following:

1- Data on patients' physical examination status stressing on the presence of extramedullary disease (hepatomegaly, splenomegaly, and lymphadenopathy) was obtained from their medical records.

2- Complete blood count (CBC) using Coulter LH 750 analyzer (Coulter Electronics, Hialeah, FL, USA), an examination of Leishman-stained peripheral blood $(\mathrm{PB})$ and bone marrow (BM) aspiration smears.

3- Routine diagnostic immunophenotyping of the BM aspirate was performed on Navios Flow cytometer (Coulter Electronics, Hialeah, FL, USA) using a panel of monoclonal antibodies including $\mathrm{B}$ cell markers: CD10, CD19, CD20; T cell markers: CD2, CD3, CD5, CD7. Myeloid markers: CD13, CD33, CD15, CD17 and monocytic marker: CD14. Common progenitor markers: CD34, HLA-DR. Cytoplasmic markers: MPO, CD79a, and CD3. Samples were considered positive for a certain marker when $\geq 20 \%$ of cells were expressing it, except for CD34 where its expression by $10 \%$ of cells was sufficient to confer positivity [14].

4- Conventional karyotyping and fluorescence in situ hybridization (FISH) in selected cases were performed.

5- Real-time polymerase chain reaction (PCR) for the detection of LEF-1 mRNA expression levels (Qiagen, Hilden, Germany).

6- All patients were followed up at day 28 from the beginning of the induction therapy to assess 
morphological remission by bone marrow examination while in 12 patients only $L E F-1$ mRNA expression levels were re-analyzed to assess the effect of therapy on expression levels.

7- Estimation of overall survival (OS) and disease-free survival (DFS) were done. Overall survival was measured from the date of diagnosis until the date of death, or the last date is known to be alive while disease-free survival was measured from the date of hematological remission until the date of death or last follow-up if alive [15].

\section{Molecular analysis of LEF-1 expression level}

Total ribonucleic acid (RNA) was isolated from whole blood samples by using the "RNeasy Serum/Plasma Kit" (Qiagen, Hilden, Germany) following manufacturer instructions. Then, mRNA of $L E F-1$ was reversibly transcribed into complementary deoxyribonucleic acid (cDNA) using QuantiTect II RT Kit (Qiagen, Hilden, Germany). The cDNA is then used for real-time PCR quantification of mature mRNA expression. LEF-1 gene expression level was amplified from mRNA using QuantiTect primer assay Hs_LEF1_1_SG QuantiTect Primer Assay (cat no 249900, ID QT00021133) (Qiagen, Germany) LEF-1 primers forward-, 5'-AGAACA CCCCGATGACGGA-3', reverse 5'-GAGGGTCCCT TGTTGTAGAGG-3'; and QuantiTect SYBR Green PCR (Kit cat no 204141) (Qiagen, Germany) and Beta-actin ( $\beta$ actin) gene expression level as housekeeper gene was amplified from mRNA using Hs_ACTB_1 SG QuantiTect Primer Assay (cat no 249900, ID QT00095431) (Qiagen, Germany). $\beta$-actin primers forward-5'TGACGTGGACATCCGCA AAG-3'; reverse 5'CTGG AAGGTGGACAGC GAGG-3'. Quantitative reverse transcription-polymerase chain reaction (RT-PCR) was performed in a total volume of $20 \mu \mathrm{L}$, containing $2 \mu \mathrm{L}$ of template cDNA, $10 \mu \mathrm{l}$ of $2 \mathrm{x}$ QuantiTect SYBR Green PCR Master Mix, $2 \mu$ 10x t Universal Primer,
$2 \mu \mathrm{l}$ 10x Quantitect Primer Assay, and $4 \mu \mathrm{l}$ RNase-free water. The real-time cycler initially was programmed as activation step $15 \mathrm{~min} 95^{\circ} \mathrm{C}$ for HotStarTaq DNA Polymerase activation. Three-step cycling is as follows: denaturation $15 \mathrm{~s} 94{ }^{\circ} \mathrm{C}$, annealing $30 \mathrm{~s}$ and $55^{\circ} \mathrm{C}$, extension $30 \mathrm{~s} 70^{\circ} \mathrm{C}$, for 40 cycles. All samples were analyzed using the 5 plex Rotor-Gene PCR Analyzer (Qiagen, Germany). Results were reported in relative quantification which is based on the expression levels of a target gene (LEF-1 mRNA) versus a reference gene ( $\beta$-actin mRNA) (Figs. 1, 2, 3).

To calculate the expression of a target gene to an adequate reference gene, calculations were done based on the comparison of a distinct cycle in real-time PCR determined by cycle threshold $(\mathrm{CT})$ values of thermal cyclers at a constant level of fluorescence [16]. After determining the cycle threshold, the $\Delta C \mathrm{~T}$ value for each sample was determined by calculating the difference between the CT value of the target gene and the $\mathrm{CT}$ value of the endogenous reference gene. This was determined for each unknown sample as well as for the calibrator sample (control subjects):

$\Delta \mathrm{CT}$ (sample) $=\mathrm{CT}$ target gene $-\mathrm{CT}$ reference gene

$\Delta \mathrm{CT}$ (calibrator) $=\mathrm{CT}$ target gene $-\mathrm{CT}$ reference gene

Next, the $\Delta \Delta C T$ value for each sample was determined by subtracting the $\Delta \mathrm{CT}$ value of the calibrator from the $\Delta \mathrm{CT}$ value of the sample: $\Delta \Delta \mathrm{CT}=\Delta \mathrm{CT}$ (sample) $-\Delta \mathrm{CT}$ (calibrator).

Finally, the normalized level of target gene expression was calculated by using the formula $2-\Delta \Delta \mathrm{CT}$ [16].

\section{Statistical analysis}

Statistical analysis was performed using SPSS V20. Quantitative data were represented as mean and standard deviation for parametric data and as median and range in non-parametric data. Qualitative data were

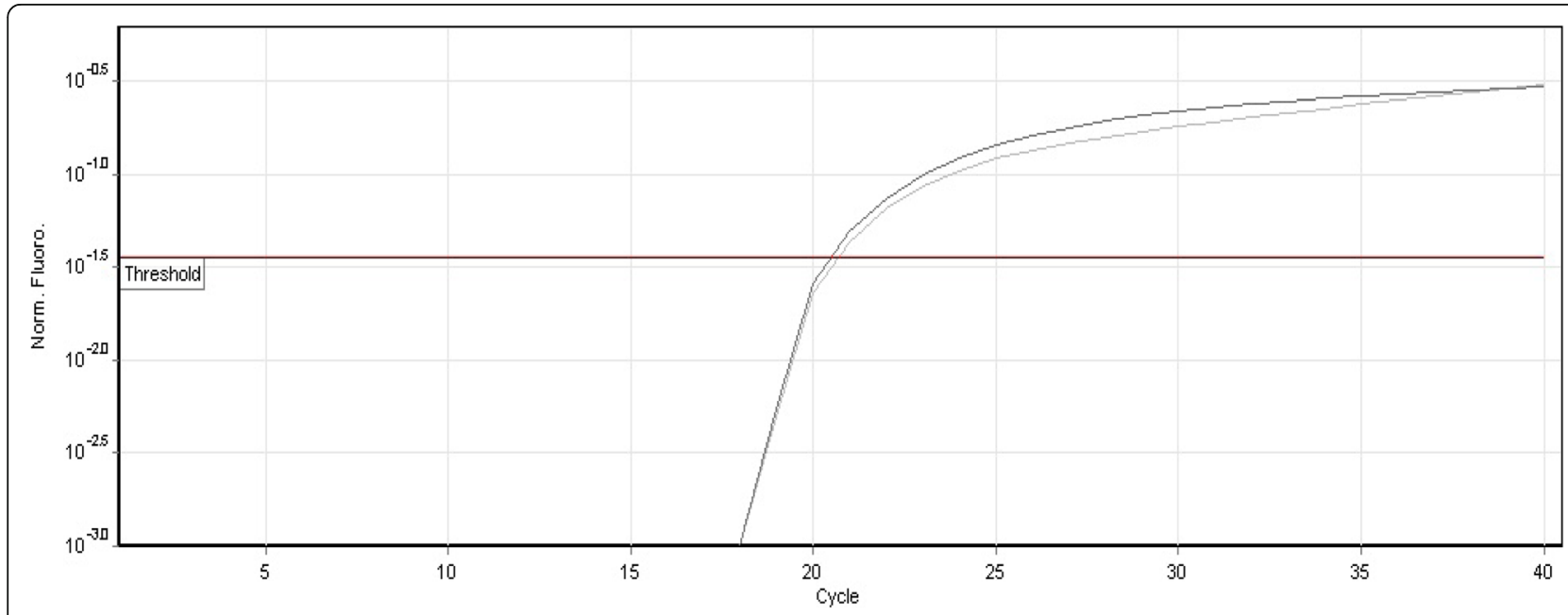

Fig. 1 Control curve with normal LEF-1 expression level, LEF1 (dark gray), ACTB (gray) 


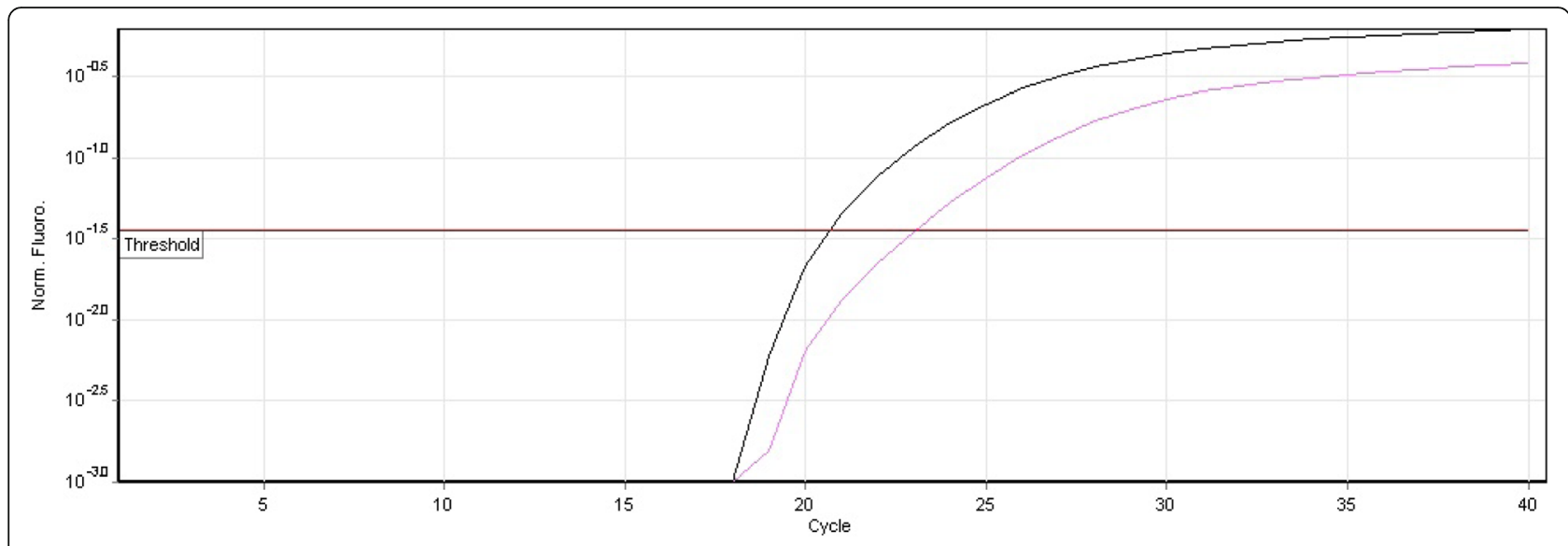

Fig. 2 Case of AML curve with high LEF-1 expression level, LEF1 (black), ACTB (violet)

represented as number and percentage. Comparisons of qualitative variables were conducted between groups using the Chi-square, and comparisons of quantitative variables were conducted between groups using the Mann Whitney for non-parametric data and student's $t$ test for parametric data, while comparisons between more than two groups with parametric distribution were done by using one way analysis of variance (ANOVA) and Kruskal-Wallis for non-parametric distributions.

Also, correlations between quantitative variables within groups were performed using the Pearson correlation coefficient. $P<0.05$ and $<0.001$ were set as statistically significant and highly significant respectively.

\section{Results}

The studied patients included 17 (56.7\%) males and 13 (43.3\%) females, with M:F ratio of 1.3:1 and a mean age of $49.4 \pm 15.0$ years. They were 21 patients $(70 \%) \leq 60$ years and $9(30 \%)>60$ years. Twelve patients $(40 \%)$ had hepatomegaly, $11(36.7 \%)$ had splenomegaly, and 8
(26.7\%) had lymphadenopathy. As regards the CBC data, total leucocytic count (TLC) showed a median and interquartile range (IQR) of $21(9.6-49.7) \times 10^{9} / \mathrm{L}$ with 7 patients $(23.3 \%)$ having TLC $>50 \times 10^{9} / \mathrm{L}$ and $23(76.6 \%)$ having TLC $<50 \times 10^{9} / \mathrm{L}$. Mean hemoglobin ( $\mathrm{Hb})$ level was $8.1 \pm 2.2 \mathrm{~g} / \mathrm{dl}$ with 24 patients $(80 \%)$ having $\mathrm{Hb}<10 \mathrm{~g} / \mathrm{dl}$, mean platelet count (Plt) was $57.4 \pm$ $36.4 \times 10^{9} / \mathrm{L}$ with 26 patients $(86.7 \%)$ having Plt count $<100 \times 10^{9} / \mathrm{L}$, and mean blast cell count in peripheral blood $(\mathrm{PB})$ was $36.8 \pm 26.8 \%$. The mean bone marrow blast count was $72.9 \pm 20.6 \%$. These demographic data were adopted from our previous study [11]. Patients were classified according to cytogenetic analysis into good, poor, and intermediate cytogenetic prognostic groups [17] besides their response to therapy at day 28 after induction by BM examination where patients who had blast cell count $<5 \%$ were considered to have hematological remission [18] (Table 1). The OS of AML patients showed a mean of $4.20 \pm 3.45$ months while DFS showed a mean of $5.9 \pm 2.5$ months.

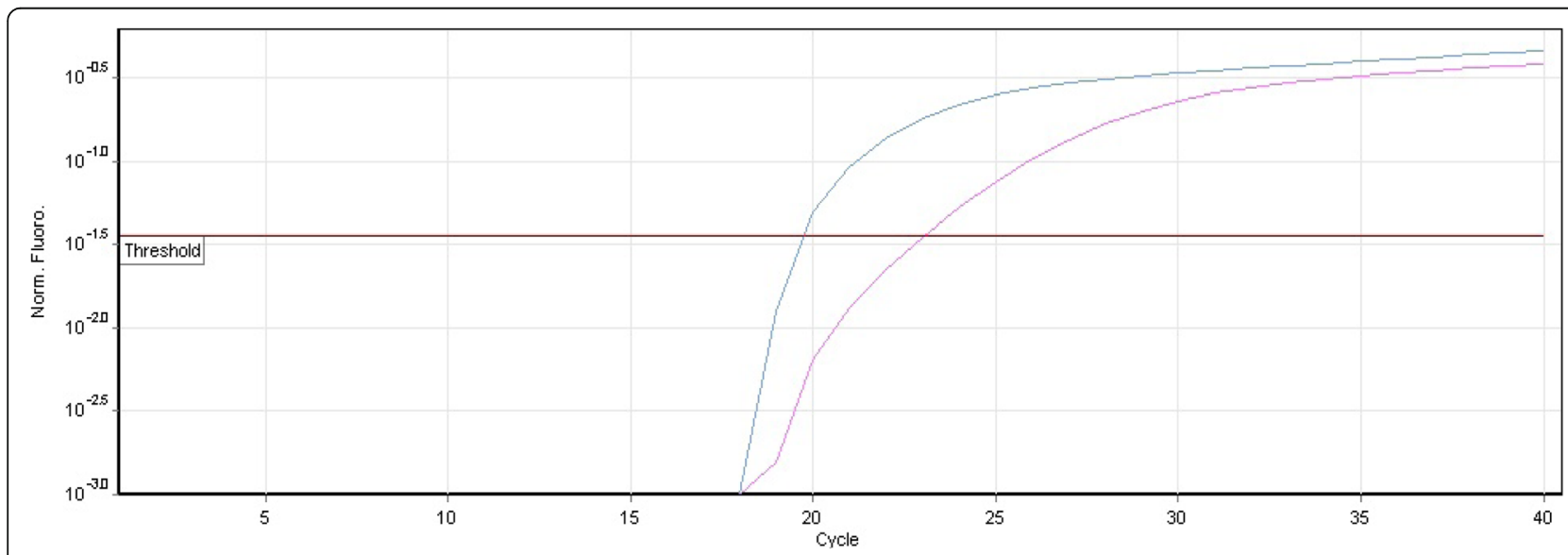

Fig. 3 Case of AML curve with low LEF-1 expression level, LEF1 (violet), ACTB (blue) 
Table 1 Cytogenetic classification and response to therapy at day 28 after induction therapy for AML patients

\begin{tabular}{llll}
\hline Parameters & & \multicolumn{2}{l}{ Patients } \\
\cline { 3 - 4 } & & $\mathrm{N}$ & $\%$ \\
\hline Cytogenetics & Normal karyotype & 15 & $50.0 \%$ \\
& $\mathrm{t}(15 ; 17)(\mathrm{q} 22 ; \mathrm{q} 12)$ & 5 & $16.7 \%$ \\
& $\mathrm{t}(8,21)(\mathrm{q} 22 ; \mathrm{q} 22)$ & 4 & $13.3 \%$ \\
& inv 16 (p13q22) & 1 & $3.3 \%$ \\
& $11 \mathrm{q} 23$ rearrangement & 2 & $6.7 \%$ \\
& Monosomy 7 & 1 & $3.3 \%$ \\
& $\mathrm{t}(9,22)(\mathrm{q} 34 ; \mathrm{q} 11)$ & 1 & $3.3 \%$ \\
& Trisomy 8 & 1 & $3.3 \%$ \\
Cytogenetics classification* & Poor & 4 & $13.3 \%$ \\
& Intermediate & 16 & $53.3 \%$ \\
& Good & 10 & $33.3 \%$ \\
Response to therapy at & Hematological remission & 9 & $30.0 \%$ \\
day 28 & Incomplete remission & 13 & $43.3 \%$ \\
& Died & 8 & $26.7 \%$
\end{tabular}

*Patients were classified as good, poor, and intermediate cytogenetic prognostic groups according to cytogenetic analysis where $t(8 ; 21)$ (q22;q22), inv16 (p13;q22), and $\mathrm{t}(15 ; 17)$ (q22;q21) were considered as good prognosis, while normal karyotype and trisomy 8 were considered intermediate prognosis, and 11q23 rearrangement, monosomy $7, t(9,22)(q 34 ; q 11)$ were considered poor prognosis by Wang and Bailey [17]

As AML patients showed to have a statistically significant higher level of LEF-1 expression compared to controls in our previous study [11], we calculated the median expression level of $L E F-1$ which was $4.82 \log _{10}$ and classified our patients according to it into high and low expression groups. Patients with values above this median were classified as high LEF-1 expression (15 patients), while those with values below this median were classified as low LEF-1 expression (15 patients). A highly statistically significant difference was found between low and high $L E F-1$ expression groups where high $L E F-1$ expression group was associated with younger age; majority of them (86.7\%) lacked the presence of hepatomegaly, had lower TLC, higher $\mathrm{Hb}$ level, higher Plt count, lower PB blast percentage, good cytogenetic prognostic group, and better response to therapy (Table 2) (Fig. 4).

Regarding patient outcome, their response at day 28 after induction therapy was assessed where high $L E F-1$ expression group had favorable treatment outcomes as 60\% (9/ 15) achieved hematological remission while none of the low LEF-1 expression patients achieved hematological remission, their outcome was either incomplete remission $7 / 15$ (46.6\%) or death $8 / 15$ (53.3\%). As well as on estimation of OS, high $L E F-1$ expression group had longer OS compared to low $L E F-1$ expression group, as all low $L E F-1$ expression patients died before reaching the first complete remission due to severe bleeding and infections. Early death occurred in $53.3 \%$ of cases while no case in the high $L E F-1$ expression group experienced early death.

In a group of 12 patients, the $L E F-1$ expression level was remeasured at day 28 after induction therapy for follow-up (unfortunately 8 patients died early during induction, and the other 10 patients, their BM samples at day 28 were too small and obtained with difficultly, and no sample was available for molecular studies). This group had $L E F-1$ expression with a mean of $10.88 \pm 4.79$ $\log _{10}$ at diagnosis and mean of $4.62 \pm 4.36 \log _{10}$ at day 28 with a highly statistically significant difference $(t=3.86$, $P=0.003$ ) (Fig. 5a). It was of note that 7 patients out of the 12 patients had hematological remission and showed a marked decrease in $L E F-1$ expression level after therapy, mean $13.25 \pm 1.81 \log _{10}$ at diagnosis, and mean of $2.09 \pm$ $0.60 \log _{10}$ at day 28 with a highly statistically significant difference ( $t=19.25, P=0.000)$ (Fig. $5 \mathrm{~b})$, while the remaining 5 patients did not achieve hematological remission and had minimal change in LEF-1 expression level at day 28 and died shortly after, mean $7.55 \pm 5.87 \log _{10}$ at diagnosis and mean of $7.53 \pm 6.04 \log _{10}$ at day 28 with no statistically significant difference $(t=0.28, P=0.791)$ (Fig. 5c) (Table 3).

The prognostic performance of $L E F-1$ expression was assessed using ROC curve analysis to obtain best cutoff for predicting the poor outcome (no achievement of remission or death). A cutoff value of $\leq 10.11 \log _{10}$ was found to have a sensitivity of $90.48 \%$ and specificity of 100\% (Fig. 6).

In our study, univariate logistic regression analysis was performed to determine the prognostic significance of $L E F-1$ expression after adjusting for the impact of other standard risk factors (age and cytogenetics) showed that the chance of the patient to achieve hematological remission was increased by 2.29 folds with every $\log _{10}$ increase in the $L E F-1$ expression level (Table 4).

\section{Discussion}

Over the past years, the impact of genetic characterization on the estimation of prognosis, treatment stratification, and development of novel targeted therapy approaches for AML has increased. Besides, several molecular markers can be used for MRD detection. Searching for new molecular prognostic markers, we studied the relevance of LEF1 expression in AML.

It was shown in our previous study [11] that LEF-1 expression levels were significantly higher in the AML patients' group compared to the control group which could be attributed to the fact that aberrant LEF-1 signaling leads to increased growth and proliferation of myeloid progenitor cells through upregulation of its target genes such as $c-m y c$ and cyclin D1 [19]. Similarly, $\mathrm{Fu}$ et al. [10], Fu et al. [20], Elhoseiny and Abdelfattah [21], and ElBaiomy et al. [22] reported similar results. Also, Petropoulos et al. [23] analyzed LEF-1 expression 
Table 2 Comparison between low and high LEF-1 expression groups regarding clinical data, laboratory data, and cytogenetic classification

\begin{tabular}{|c|c|c|c|c|c|c|c|c|}
\hline \multirow[t]{3}{*}{ Parameters } & & \multicolumn{4}{|l|}{ LEF1 } & \multirow{2}{*}{\multicolumn{3}{|c|}{ Test of significance }} \\
\hline & & \multicolumn{2}{|c|}{$\begin{array}{l}\text { Low expression }<4.82 \log _{10} \\
\boldsymbol{N}=15\end{array}$} & \multicolumn{2}{|c|}{$\begin{array}{l}\text { High expression }>4.82 \log _{10} \\
\boldsymbol{N}=15\end{array}$} & & & \\
\hline & & $\overline{M e a n} / \boldsymbol{N}$ & $\mathrm{SD} / \%$ & Mean $/ \boldsymbol{N}$ & $\mathrm{SD} / \%$ & Test & $\boldsymbol{p}$ value & Sig \\
\hline Age & & 61.0 & \pm 10.2 & 37.7 & \pm 8.4 & $t=6.82$ & $<0.001$ & $\mathrm{HS}$ \\
\hline \multirow[t]{2}{*}{ Sex } & Male & 9 & $60.0 \%$ & 8 & $53.3 \%$ & $\mathrm{~F}$ & 0.713 & NS \\
\hline & Female & 6 & $40.0 \%$ & 7 & $46.7 \%$ & & & \\
\hline \multirow[t]{2}{*}{ Hepatomegaly } & No & 5 & $33.3 \%$ & 13 & $86.7 \%$ & $x^{2}=8.8$ & 0.003 & HS \\
\hline & Yes & 10 & $66.7 \%$ & 2 & $13.3 \%$ & & & \\
\hline \multirow[t]{2}{*}{ Splenomegaly } & No & 7 & $46.7 \%$ & 12 & $80.0 \%$ & $x^{2}=3.5$ & 0.058 & NS \\
\hline & Yes & 8 & $53.3 \%$ & 3 & $20.0 \%$ & & & \\
\hline \multirow[t]{2}{*}{ Lymph-adenopathy } & No & 10 & $66.7 \%$ & 12 & $80.0 \%$ & $\mathrm{~F}$ & 0.682 & NS \\
\hline & Yes & 5 & $33.3 \%$ & 3 & $20.0 \%$ & & & \\
\hline $\mathrm{Hb}(\mathrm{g} / \mathrm{dL})$ & & 6.9 & \pm 2.2 & 9.4 & \pm 1.4 & $t=-3.7$ & 0.001 & HS \\
\hline $\operatorname{TLC}\left(\times 10^{9} / \mathrm{L}\right)$ & & 71.0 & \pm 53.1 & 9.5 & \pm 5.5 & $t=4.46$ & 0.001 & HS \\
\hline PLT $\left(\times 10^{9} / \mathrm{L}\right)$ & & 32.8 & \pm 14.2 & 82.0 & \pm 35.2 & $t=-5.0$ & $<0.001$ & HS \\
\hline PB blast (\%) & & 52.1 & \pm 28.3 & 19.5 & \pm 10.6 & $t=4.19$ & 0.001 & HS \\
\hline BM blast (\%) & & 70.9 & \pm 21.2 & 74.9 & \pm 20.5 & $t=-0.5$ & 0.603 & NS \\
\hline \multirow[t]{3}{*}{ Cytogenetics classification } & Poor & 4 & $26.7 \%$ & 0 & $0.0 \%$ & $\mathrm{~F}$ & 0.015 & S \\
\hline & Intermediate & 9 & $60.0 \%$ & 7 & $46.7 \%$ & & & \\
\hline & Good & 2 & $13.3 \%$ & 8 & $53.3 \%$ & & & \\
\hline \multirow[t]{3}{*}{ Response to therapy at day 28} & Hematological Remission & 0 & $0.0 \%$ & 9 & $60.0 \%$ & $\mathrm{~F}$ & $<0.001$ & HS \\
\hline & Incomplete Remission & 7 & $46.7 \%$ & 6 & $40.0 \%$ & & & \\
\hline & Died & 8 & $53.3 \%$ & 0 & $0.0 \%$ & & & \\
\hline Overall survival (months) & & 2.75 & \pm 2.00 & 6.40 & \pm 2.59 & $t=4.52$ & $<0.001$ & HS \\
\hline Disease free survival (months) & & - & - & 5.9 & \pm 2.5 & & & \\
\hline
\end{tabular}

$N$ number, $H b$ hemoglobin, TLC total leucocytic count, $P B$ peripheral blood, $B M$ bone marrow, LEF-1 lymphoid enhancer binding factor 1, $t$ student's $t$ test, $F$ Fisher's exact test, $X^{2}$ Chi square test, $S$ significant, $H S$ highly significant, NS non-significant

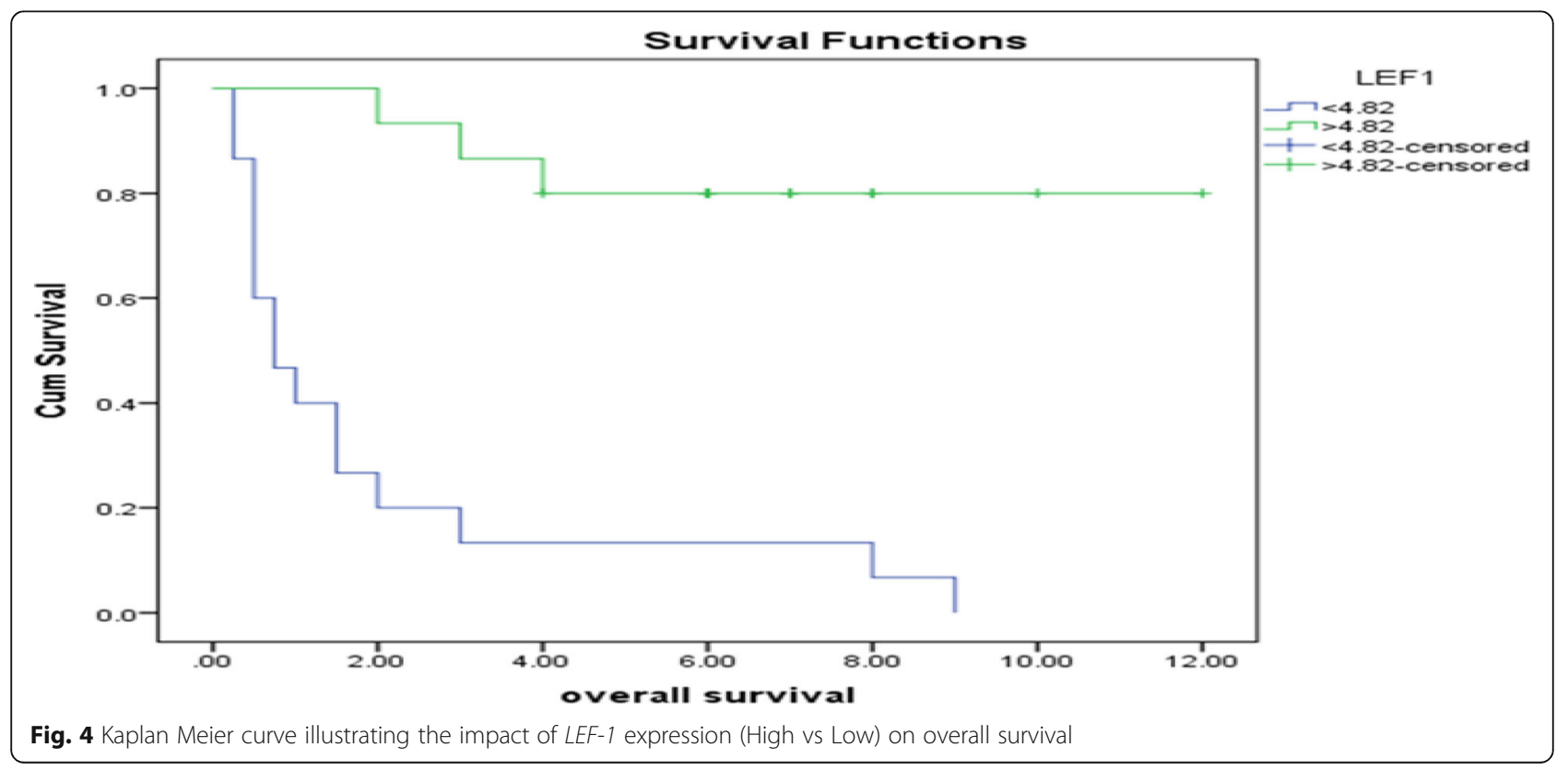




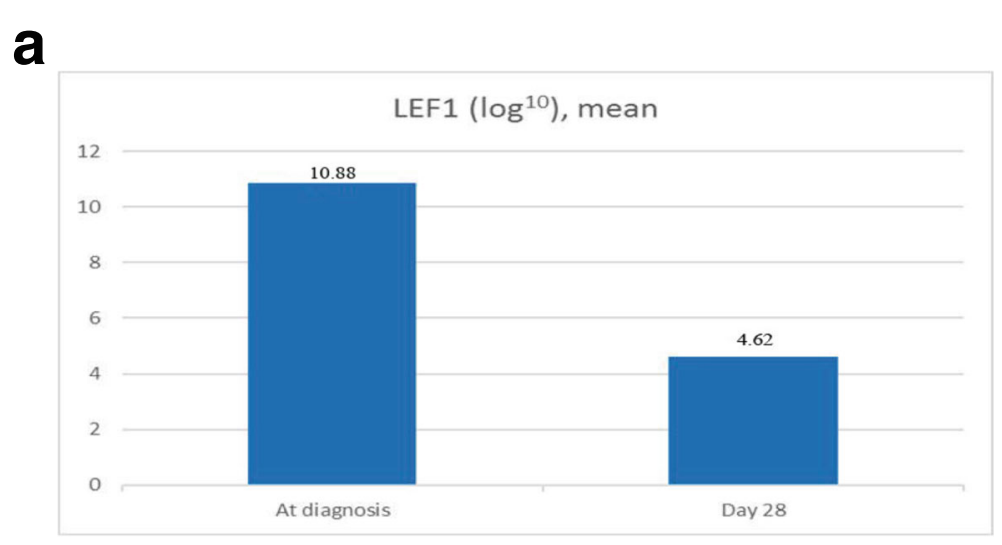

b

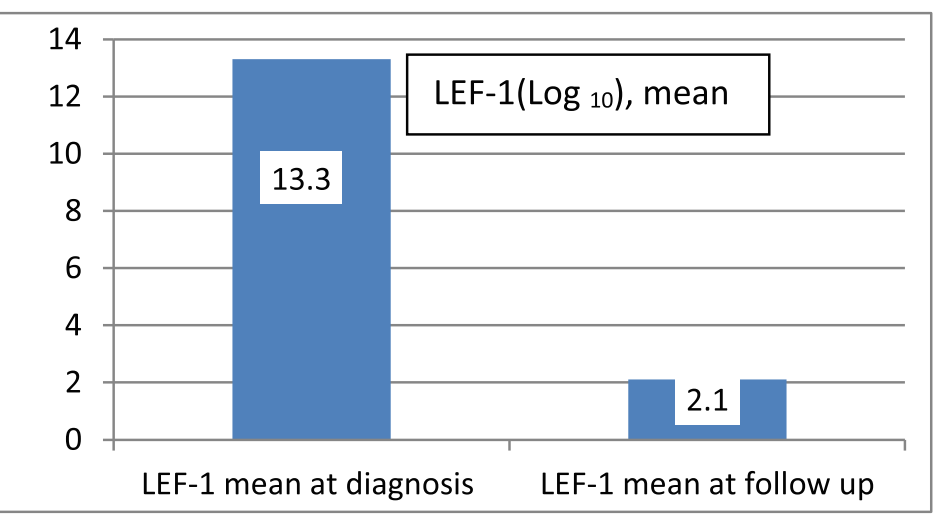

C

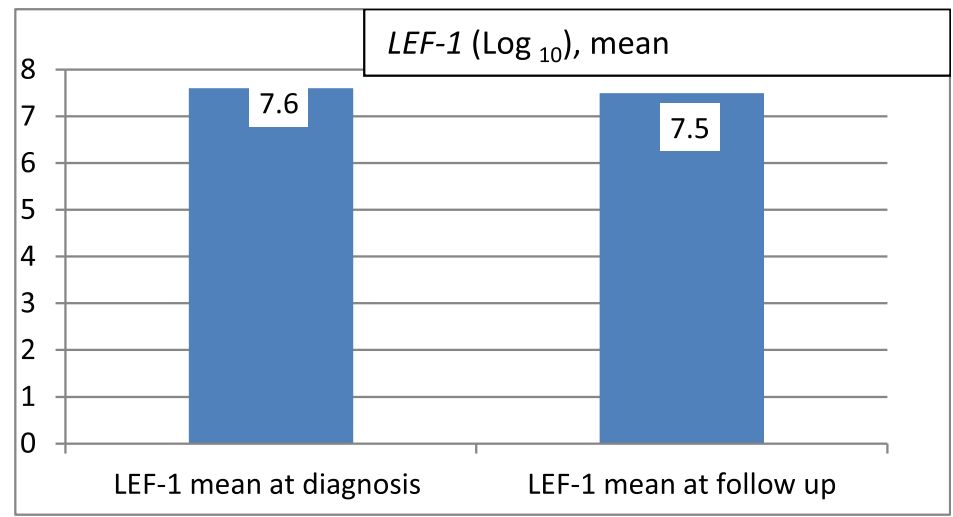

Fig. 5 a Comparison between LEF-1 expression at diagnosis and day 28 after therapy in a group of 12 patients. b Comparison between LEF-1 expression at diagnosis and day 28 after therapy in a group of 7 out 12 patients whom LEF-1 level showed significant decrease at day 28. $\mathbf{c}$ Comparison between LEF-1 expression at diagnosis and day 28 after therapy in a group of 5 out 12 patients whom LEF-1 level did not show any significant change at day 28

in normal $\mathrm{BM}$ and human leukemias and reported that leukemic samples, as well as normal BM samples, were positive for $L E F-1$ expression, but acute lymphoblastic leukemia (ALL) samples showed about 13-fold higher relative expression of $L E F-1$ compared with AML possibly reflecting the higher expression of the transcription factor in lymphoid tissue.
Aiming to better elucidate the prognostic significance of LEF-1 expression in our patients' group, we thought to remeasure $L E F-1$ expression level at day 28 of induction therapy in addition to assessing their remission status and following them for 1 year to be able to assess the effect of $L E F-1$ expression on the survival. We classified our patients into low and high expression groups 
Table 3 Clinical and laboratory characteristics of the group of patients (12 patients) where LEF-1 was remeasured at day 28

\begin{tabular}{|c|c|c|c|c|c|c|c|c|c|c|c|c|}
\hline Case $(\boldsymbol{N})$ & $\begin{array}{l}\text { Age } \\
\text { (years) }\end{array}$ & Sex & Cytogenetic & $\mathrm{Hb}(\mathrm{g} / \mathrm{dl})$ & $\mathrm{TLC} \times 10^{9} / \mathrm{L}$ & Plt $\times 10^{9} / \mathrm{L}$ & PB blast \% & BM blast \% & $\begin{array}{l}L E F-1 \\
\left(\log _{10}\right)\end{array}$ & $\begin{array}{l}\text { Follow-up LEF-1 } \\
\left(\log _{10}\right)\end{array}$ & Fate & $\begin{array}{l}\text { Response } \\
\text { (D 28) }\end{array}$ \\
\hline 1 & 32 & $\mathrm{~F}$ & $t(15 ; 17)$ & 9.1 & 5.8 & 114 & 12 & 70 & 13.93 & 3.21 & Alive & $H R$ \\
\hline 2 & 30 & $\mathrm{~F}$ & $\mathrm{t}(8,21)$ & 10.9 & 9.6 & 111 & 30 & 80 & 15.51 & 2.11 & Alive & $\mathrm{HR}$ \\
\hline 3 & 50 & $\mathrm{~F}$ & $\mathrm{t}(8,21)$ & 9.1 & 12.3 & 80 & 16 & 88 & 11.51 & 1.74 & Alive & $H R$ \\
\hline 4 & 32 & M & $\mathrm{t}(8,21)$ & 8.6 & 10 & 80 & 24 & 56 & 11.39 & 1.36 & Alive & $\mathrm{HR}$ \\
\hline 5 & 47 & M & Normal & 9 & 6 & 90 & 15 & 80 & 12.13 & 12.28 & Died & $\mathbb{I R}$ \\
\hline 6 & 28 & $\mathrm{~F}$ & Normal & 13.1 & 3.7 & 77 & 8 & 85 & 13.07 & 13.31 & Died & $\mathbb{I R}$ \\
\hline 7 & 36 & M & Normal & 8.6 & 12 & 88 & 9 & 99 & 10.11 & 9.95 & Died & $\mathbb{I R}$ \\
\hline 8 & 53 & $\mathrm{~F}$ & $t(15,17)$ & 9 & 4 & 156 & 30 & 95 & 15.39 & 2.51 & Alive & $\mathrm{HR}$ \\
\hline 9 & 30 & M & $t(15,17)$ & 10 & 4 & 80 & 35 & 30 & 13.52 & 1.82 & Alive & $H R$ \\
\hline 10 & 37 & M & $t(15,17)$ & 11 & 5 & 90 & 40 & 60 & 11.53 & 1.93 & Alive & $H R$ \\
\hline 11 & 64 & M & $11 q 23$ & 6.4 & 83.7 & 28 & 90 & 91 & 0.7 & 0.5 & Died & $\mathbb{I R}$ \\
\hline 12 & 68 & M & $11 q 23$ & 6.6 & 70.8 & 16 & 40 & 67 & 1.78 & 1.62 & Died & $\mathbb{R}$ \\
\hline
\end{tabular}

$N=$ number, $F$ female, $M$ male, $H b$ hemoglobin, $T L C$ total leucocytic count, $P B$ peripheral blood, $B M$ bone marrow, $L E F-1$, lymphoid enhancer binding factor $1, H R$ hematological remission, IR incomplete remission

according to $L E F-1$ median expression level (4.82 $\left.\log _{10}\right)$; a similar approach was adopted by several studies including Metzeler et al. [24], Fu et al. [10], Albano et al. [25], and Jia et al. [1].

The high LEF-1 expression group was younger in age and the majority lacked organomegaly, yet a significant difference was noticed regarding hepatomegaly between high and low expression groups. Similarly, Albano et al.
[25] showed a trend toward an association between high LEF-1 expression and lower median age. Also, Elhoseiny and Abdelfattah [21] reported that hepatosplenomegaly was more represented in low $L E F-1$ expression group, and the difference was statistically significant.

As for the $\mathrm{CBC}$ and $\mathrm{BM}$ data, high $L E F-1$ expression group had lower TLC, PB blast percentage, higher $\mathrm{Hb}$ level, and PLT counts in addition to a trend to lower

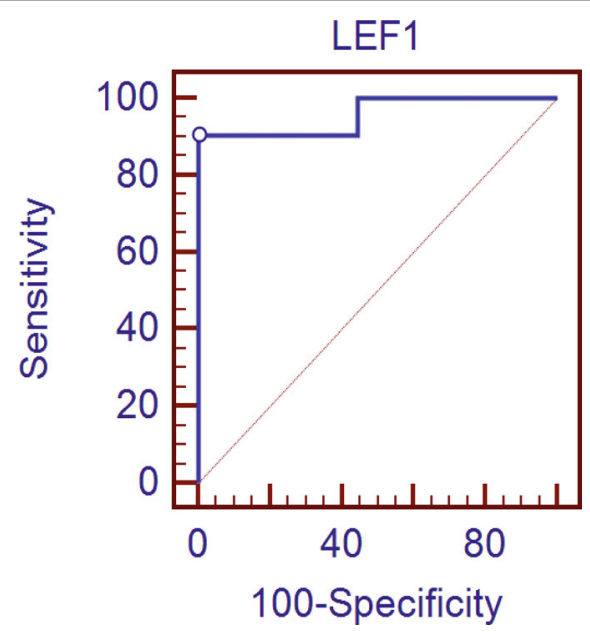

\begin{tabular}{|l|c|c|c|c|c|c|c|c|}
\hline AUC & $\mathbf{9 5} \%$ CI & $\begin{array}{c}\text { Cutoff } \\
\text { point }\end{array}$ & Sensitivity & Specificity & PPV & NPV & p value & sig. \\
\hline 0.958 & 0.814 to 0.998 & $\begin{array}{l}\leq 10.11 \\
\log _{10}\end{array}$ & 90.48 & 100 & 100 & 81.8 & 0.0001 & HS \\
\hline
\end{tabular}

Fig. 6 ROC curve analysis of LEF-1 expression predicting poor outcome. AUC, area under the curve; $\mathrm{Cl}$, confidence interval; PPV, positive predictive value; NPV, negative predictive value; sig, significance; HS, highly significant 
Table 4 Univariate logistic regression analysis of $L E F-1$ to predict hematological remission

\begin{tabular}{llll}
\hline Parameter & OR $(95 \% \mathrm{Cl})$ & $p$ value & Sig. \\
\hline LEF-1 expression & $2.29(1.001-5.27)$ & 0.049 & S \\
\hline
\end{tabular}

LEF-1 lymphoid enhancer binding factor $1, O R$ odds ratio, $\mathrm{Cl}$ confidence interval, sig. significance

mean BM blast percentage. In agreement with these results, Metzeler et al. [24] found that cytogenetically normal (CN) AML patients with low LEF-1 expression had higher TLC and BM blast percentage. Also, Albano et al. [25] who studied LEF-1 expression in APL suggested an explanation for the association of high $L E F-1$ expression and low TLC by their determination of 9 differentially expressed, up-modulated genes (ETS1, FAIM3, CCR7, IL7R, LCK, IL2RB, ITK, RASGRP1, TRBC1) in this group of patients, the majority of which is involved in the regulation of apoptosis (FAIM3, IL2RB, LCK, ETS1); moreover, it was of note that 6 (CCR7, IL7R, LCK, IL2RB, $I T K, R A S G R P 1)$ of the 9 genes were included among the signature of the 200 genes showing the strongest absolute correlation with $L E F-1$ expression levels in CN-AML [24].

Moreover, $L E F-1$ is a crucial transcription factor in neutrophilic granulopoiesis. $L E F-1$ expression is low or absent in patients with severe congenital neutropenia, leading to downregulation of CEBPA and a block of neutrophilic differentiation [2]. Thus, low LEF-1 expression may also contribute to the differentiation block in myelodysplastic syndrome (MDS) and AML blasts, as reflected by the higher TLC and blast percentages in LEF-1 low CN-AML and MDS [26].

For further elaboration of the significance of $L E F-1$ expression, we analyzed it concerning cytogenetic prognostic groups. There was a significant difference between patients in the high and low expression groups, where high $L E F-1$ expression group were either in the good or intermediate cytogenetic prognostic groups, and none was in the poor cytogenetic prognostic group, while the majority of low $L E F-1$ expression group were in the intermediate or poor cytogenetic prognostic groups. Also, all the 7 patients in whom $L E F-1$ was remeasured at day 28 and showed significant decrease in $L E F-1$ expression level and had good outcome were in the good or intermediate cytogenetic prognostic group. This might point to a relation with these cytogenetic abnormalities. In agreement, Albano et al. [25] and Fu et al. [10] reported that deregulation of $L E F-1$ contributed to the pathophysiology of AML with the most frequent balanced translocations such as $t(8,21)$ and $t(15,17)$. Inline, Müller-Tidow et al. [27] explained the association of good prognostic cytogenetic abnormalities and $L E F-1$ high expression by their finding that $P M L-R A R \alpha$ fusion gene (but also PLZF-RAR $\alpha$ and AML1-ETO) can induce plakoglobin $(\gamma$-catenin) expression in cell lines as well as in primary patient samples, resulting in transcriptional activation of $L E F-1$. Also, it has been observed that $L E F$ 1 cross talks with the Notch signaling pathway as Jagged1 (JAG1) is a downstream target gene of $L E F-1$ and is also the ligand of Notch [28]. Thus, LEF-1 can regulate the expression of JAG1 on the cytomembrane [29], and it is known that JAG1 is more strongly expressed in APL than in other AML subtypes. Further evidence of the relationship between $L E F-1$ and Notch signaling is the finding that the Notch intracellular domain (NICD) has been identified as a coactivator of $L E F-1$; thus, the effects of Notch on LEF-1 activity are direct and not due to modulation of components of the Wnt signaling cascade [30]. High LEF-1 expression group had favorable treatment outcomes and a better OS. These findings were in agreement with Metzeler et al. [24] who confirmed the association of high LEF-1 status with longer relapse-free survival (RFS), OS, and event-free survival (EFS) in multivariable analyses adjusting for the most important clinical and molecular prognosticators in CN-AML. Furthermore, they performed genome-wide gene expression profiles to identify biologic pathways that are associated with $L E F-1$ expression in CN-AML and discovered that high $L E F-1$ expression patients showed upregulation of gene sets related to $\mathrm{T}$ lymphoid differentiation. On the other hand, gene sets related to cell proliferation, DNA replication, and DNA repair were downregulated, which might contribute to their favorable outcomes.

Fu et al. [10] suggested a scoring system based on $L E F-1$ level, and mutation status of FLT3-ITD or NPM1 predict the outcome for AML patients with intermediate-risk cytogenetics as their data indicated that high LEF-1 expression predicts a significantly better OS for patients with intermediate-risk cytogenetics, although no significant difference was found in survival according to $L E F-1$ level among total patients with AML.

Moreover, Albano et al. [25] has shown that LEF-1 expression is a strong independent OS prognostic factor in APL, as high LEF-1 group had a better outcome in terms of OS, even upon studying on two different age groups (> 60 and < 60 years). Also, Elhoseiny and Abdelfattah [21] demonstrated favorable treatment outcomes for CN-AML with high LEF-1 expression as they had better OS; cumulative survival at 3 years was $87.5 \%$ in high $L E F-1$ compared to only $47.5 \%$ in low $L E F-1$ patients; the difference was statistically significant $(P=0.001)$. Hazards ratio was 0.19 (95\% CI 0.06-0.57) which means that the high level of $L E F-1$ gene is $81 \%$ protective against the hazard of death.

Early death occurred in $53.3 \%$ of cases while no case in the high $L E F-1$ expression group experienced early death. This goes with the poor prognostic significance of this group in addition to the association with other 
adverse prognostic features. Albano et al. [25] confirmed that these were early death occurred also only in their low LEF-1 expression group including $23 \%$ of their cases.

We have been able to re-analyze $L E F-1$ expression level in 12 patients after induction therapy, where a dramatic decrease in the expression level was encountered in $7 / 12(58.3 \%)$ patients who had achieved hematological remission also. This was in agreement with $\mathrm{Fu}$ et al. [10] who analyzed the impact of therapy on the $L E F-1$ level in eight patients who achieved a complete remission following successful induction therapy.

In univariate logistic regression analysis for hematological remission achievement, high $L E F-1$ patients showed an odds ratio of 2.29 fold increase toward achieving hematological remission. This was supported by Metzeler et al. [24] who stated that high LEF-1 patients showed a trend toward higher odds for the achievement of remission $(P=0.08$; odds ratio 1.82 ; $95 \%$ confidence interval $0.94-$ $3.55)$ and found that the only factors significantly associated with a higher chance of reaching complete remission were younger age $(P=0.04)$ and presence of an NPM1 mutation. This was contradictory to $\mathrm{Fu}$ et al. [10] who reported that their multivariable analysis failed to identify LEF-1 as an independent prognostic factor.

\section{Conclusion}

We are aware of the limitations of our study being singlecenter study, a small number of participants, heterogeneity of the study group, and lack of association with the key prognostic molecular markers commonly tested and used along with cytogenetics for AML risk stratification, including FLT3, NPM1, CEBPA, TP53, etc. and no longer follow-up period, which may be inadequate to determine and explain potentially unique influences on treatment outcomes. Despite these limitations, our study tried to propose a cutoff value for $L E F-1$ which requires further studies to establish its usage in risk classifications of AML. LEF-1 confers a promising target for therapy as small molecule Wnt pathway inhibitors were found to be cytotoxic for AML blasts [31].

\footnotetext{
Abbreviations

ALL: Acute lymphoblastic leukemia; AML: Acute myeloid leukemia; ANOVA: One way analysis of variance; APL: Acute promyelocytic leukemia; ATRA: All-trans-retinoic acid; ATO: Arsenic trioxide; BM: Bone marrow; CBC: Complete blood count; CN: Cytogenetically normal; CT: Cycle threshold; cDNA: Complementary deoxyribonucleic acid; D.F.S: Disease-free survival; EFS: Event-free survival; ESMO: European Society for Medical Oncology; FLAG: Fludarabine, cytarabine, and G-CSF; FISH: Fluorescence in situ hybridization; HAM: High dose cytarabine and mitoxantrone; Hb: Hemoglobin level; IQR: Interquartile range; JAG1: Jagged1; LEF1: Lymphoid enhancer-binding factor 1; M: F: Male: female; MRD: Minimal residual disease; MDS: Myelodysplastic syndrome; NICD: Notch intracellular domain; O.S: Overall survival; PB: Peripheral blood; PIt: Mean platelet count; RNA: Ribonucleic acid; RFS: Relapse-free survival; TLC: Total leucocytic count; WHO: World Health Organization; Wnt: Wingless-type
}

\section{Acknowledgements}

Not applicable.

\section{Authors' contributions}

NS: Study concept and supervision, protocol design, technical support in practical part and critical revision of the manuscript for intellectual content. BF: Helped in the protocol design, study supervision, analysis of data, and preparing the initial draft of the manuscript and the final revision. DE: Helped in the analysis of data, study supervision and the final revision. RG: Helped in the protocol design, study supervision, analysis of data, and preparing the initial draft of the manuscript and the final revision. NE: Helped in collecting data, its analysis, and the final revision. SP: Helped in the protocol design, collecting data, analysis of data, preparing the initial draft of the manuscript, material support and final revision. Finally, all authors have read and approved the manuscript.

\section{Funding}

Not applicable.

\section{Availability of data and materials}

All data generated or analyzed during this study are included in this published article, and its supplementary information files are available from the corresponding author on reasonable request.

\section{Ethics approval and consent to participate}

The protocol of study was approved by the institutional review board and in accordance with the stipulations of the local ethical and scientific committees of Ain-Shams University Hospitals, Faculty of medicine (reference number of the ethical committee FWA000017585), and the procedures respected the ethical standards in the Helsinki declaration of 1964. Written informed consents were obtained from patients to use their samples in this study.

Consent for publication

Not applicable

\section{Competing interests}

The authors declare that they have no competing interests.

\section{Author details}

${ }^{1}$ Clinical and Chemical Pathology, Hematology unit, Faculty of Medicine, Ain Shams University, Cairo, Egypt. ${ }^{2}$ Oncology Diagnostic Unit, Biomedical Research Department, Faculty of Medicine, Ain Shams University, Cairo, Egypt.

Received: 24 March 2020 Accepted: 11 June 2020

Published online: 22 July 2020

\section{References}

1. Jia M, Zhao H, Shen H, Cheng Y, Luo Z (2015) Li, et al. overexpression of lymphoid enhancer-binding factor-1 (LEF1) is a novel favourable prognostic factor in childhood acute lymphoblastic leukaemia. Int Jnl Lab Hem 37:631-640

2. Skokowa J, Welte K (2007) LEF-1 is a decisive transcription factor in neutrophil granulopoiesis. Ann N Y Acad Sci 1106:143-151

3. Pehlivan M, Caliskan C, Yuce Z, Sercan H (2017) Forced expression of Wnt antagonists SFRP1 and WIF1 sensitizes chronic myeloid leukaemia cells to tyrosine kinase inhibitors. Tumour Biol 39(5):1010428317701654. https://doi. org/10.1177/1010428317701654

4. Pehlivan M, Çalışkan C, Yüce Z, Sercan H $(2018,69)$ Secreted Wnt antagonists in leukaemia: a road yet to be paved. Leukaemia research:24-30

5. Aly R, Yousef A (2015) Prognostic significance of lymphoid enhancerbinding factor-1 expression in Egyptian adult B-acute lymphocytic leukaemia patients. Turk J Hematol 32:15-20

6. Menter T, Trivedi P, Ahmad R, Flora R, Dirnhofer S, Tzankov A et al (2017) Diagnostic utility of lymphoid enhancer binding factor 1 immunohistochemistry in small B-cell lymphomas. Am J Clin Pathol 147: 292-300

7. Tandon B, Peterson L, Gao J, Nelson B, Ma S, Rosen S et al (2011) Nuclear overexpression of lymphoid-enhancer-binding factor 1 identifies chronic 
lymphocytic leukaemia/small lymphocytic lymphoma in small B-cell lymphomas. Mod Pathol 24(11):1433-1443

8. ElSourdy M, Ayad M, Fayad A, Youssef S (2019) Lymphoid enhancer factor 1 gene expression in comparison to other prognostic markers in adult Bacute lymphoblastic leukaemia. Egypt J Haematol 44:40-47

9. Gutierrez A, Sanda T, Ma W, Zhang J, Grebliunaite R, Dahlberg S et al (2010) Inactivation of LEF1 in T-cell acute lymphoblastic leukaemia. Blood. 115: 2845-2851

10. Fu Y, Zhu H, Wu W, Xu J, Chen T, Xu B et al (2014) Clinical significance of lymphoid enhancer-binding factor 1 expression in acute myeloid leukaemia. Leukaemia \& Lymphoma 55:371-377

11. Shalaby N, Eissa D, Farweez B, Pessar S, Galal R (2018) Clinical significance of lymphoid enhancer-binding factor 1 (LEF-1) expression in acute myeloid leukemia. The Egyptian Journal of Hospital Medicine 72(6):4686-4692. https://doi.org/10.12816/EJHM.2018.9841

12. Arber D, Brunning R, Orazi A, Brunning R, Le Beau M, Porwit A. Acute myeloid leukaemia and related precursor neoplasms. In: WHO Classification of Tumours of Haematopoietic and Lymphoid Tissues, Steven H Swerdlow, Elias Campo, Nancy Lee Harris, Elaine S Jaffe, Stefano A Pileri, Harald Stein, Jurgen Thiele, (eds) revised fourth edition. International Agency for Research on Cancer (IARC) 69008 Lyon, France. 2017. Chapter 8: 129-170.

13. Fey M, Buske C, on behalf of the ESMO Guidelines Working Group (2013) Acute myeloblastic leukaemias in adult patients: ESMO clinical practice guidelines for diagnosis, treatment and follow-up. Ann Oncol 24(Suppl 6): vil38-vil43

14. Matutes E, Morillo R and Morillo A. Immunophenotypic analysis of acute myeloid leukaemia. In: Dacie and Lewis Practical hematology (eds. Lewis S. Bain B. and Bates I), 11th edition, 2012. Elsevier, Churchill, Livingstone, China, Chapter 16: 362-4.

15. El Ghafar A, El-Sakhawy Y, Safwat N, Ismail H (2018) Cytogenetic analysis of acute myeloid leukaemia with $t(8 ; 21)$ : its clinical correlation with loss of $X$ chromosome and Del (9q). Journal of Applied Hematology 9:51-58

16. Page R, Stromberg A (2011) Linear methods for analysis and quality control of relative expression ratios from quantitative real-time polymerase chain reaction experiments. Sci World J 11:1383-1393

17. Wang M, Bailey N (2015) Acute myeloid leukaemia genetics: risk stratification and implications for therapy. Archives of pathology \& laboratory medicine 139:1215-1223

18. Øvlisen A, Oest A, Bendtsen $M, B æ c h ~ J$, Johansen P, Lynggaard $L$ et al (2018) Stringent or non-stringent complete remission and prognosis in acute myeloid leukaemia: a Danish population-based study. Blood advances 2:559-564

19. Zarkou V, Galaras A, Giakountis A, Hatzis P (2018) Crosstalk mechanisms between the WNT signalling pathway and long non-coding RNAs. Noncoding RNA Research 3:42-53

20. Fu Y, Zhu H, Wu W, Xu J, Shen $W, X u W$, et al. The expression of LEF1 gene and its clinical significance in Chinese patients with acute myeloid leukemia. Blood .2012: 120 (21): 4621.https://doi.org/https://doi.org/10.1182/blood. V120.21.4621.

21. Elhoseiny S, Abdelfattah R (2016) Overexpression of lymphoid enhancerbinding factor-1 (lef1) predict favorable outcome in cytogenetically normal acute myeloid leukaemia (CN-AML). Int J Adv Res 4(3):1532-1547

22. ElBaiomy M, Aref S, El Zaafarany M, Atwa S, Akl T, El-Beshbishi W, et al. Prognostic impact of lymphoid enhancer factor 1 expression and serum galectin.3 in Egyptian AML patients. Advances in Hematology. 2019, Article ID 2352919. https://doi.org/https://doi.org/10.1155/2019/2352919.

23. Petropoulos K, Arseni N, Schessl C, Stadler C, Rawat V, Deshpande A et al (2008) A novel role for Lef-1, a central transcription mediator of Wnt signalling, in leukemogenesis. J Exp Med 205:515-522

24. Albano et al. confirmed that early deaths ocurred also only in their low LEF1 expression group including 23\%of their cases. Blood. 120:2118-2126

25. Albano F, Zagaria A, Anelli L, Orsini P, Minervini C, Impera L et al (2014) Lymphoid enhancer-binding factor-1 (LEF1) expression as a prognostic factor in adult acute promyelocytic leukaemia. Oncotarget. 5:649-658

26. Pellagatti A, Marafioti T, Paterson J, Malcovati L, Della Porta M et al (2009) Marked downregulation of the granulopoiesis regulator LEF1 is associated with disease progression in the myelodysplastic syndromes. Br J Haematol 146(1):86-90

27. Müller-Tidow C, Steffen B, Cauvet T, Tickenbrock L, Ji P, Diederichs S et al (2004) Translocation products in acute myeloid leukaemia activate the Wnt signalling pathway in hematopoietic cells. Mol Cell Biol 24:2890-2904
28. Zhang Y, Yu J, Shi C, Huang Y, Wang Y, Yang T et al (2013) Lef1 contributes to the differentiation of bulge stem cells by nuclear translocation and crosstalk with the notch signalling pathway. Int J Med Sci 10:738-746

29. Ross D, Kadesch $T$ (2001) The notch intracellular domain can function as a coactivator for LEF-1. Mol Cell Biol 21(22):7537-7544

30. Shih L, Kuo M, Liang D, Huang C, Lin T, Wu J et al (2003) Internal tandem duplication and Asp835 mutations of the FMS-like tyrosine kinase 3 (FLT3) gene in acute promyelocytic leukaemia. Cancer. 98:1206-1216

31. Minke K, Staib P, Puetter A, Gehrke I, Gandhirajan R, Schlösser A et al (2009) Small molecule inhibitors of WNT signalling effectively induce apoptosis in acute myeloid leukaemia cells. Eur J Haematol 82:165-175

\section{Publisher's Note}

Springer Nature remains neutral with regard to jurisdictional claims in published maps and institutional affiliations.

\section{Submit your manuscript to a SpringerOpen ${ }^{\circ}$ journal and benefit from:}

- Convenient online submission

- Rigorous peer review

- Open access: articles freely available online

- High visibility within the field

- Retaining the copyright to your article

Submit your next manuscript at $\boldsymbol{\nabla}$ springeropen.com 